\title{
DESENVOLVIMENTO DE PLÂNTULAS DE PESSEGUEIRO 'OKINAWA' INOCULADAS COM MICORRIZAS ARBUSCULARES ISOLADAS DE POMARES DE PESSEGUEIROS E DE VINHEDOS ${ }^{1}$
}

\author{
JOSÉ LUÍS DA SILVA NUNES², PAULO VITOR DUTRA DE SOUZA³, \\ GILMAR ARDUINO BETTIO MARODIN ${ }^{3}$, JOSÉ CARLOS FACHINELLO ${ }^{4}$
}

RESUMO - O objetivo deste trabalho foi avaliar a influência das espécies de micorrizas arbusculares (MA) Glomus clarum e Glomus etunicatum isoladas de pomares de pessegueiros e de vinhedos sobre o crescimento vegetativo, conteúdo de macronutrientes e substâncias de reserva em plantas do porta-enxerto de pessegueiro cv. Okinawa. O experimento foi conduzido em telado, com delineamento experimental de blocos casualizados, em parcelas subdivididas, com 15 plantas por parcela e quatro repetições. A inoculação dos isolados de MA favoreceu a absorção de nitrogênio, fósforo e potássio, contribuindo para que as plantas inoculadas apresentassem as maiores respostas em altura, diâmetro, área foliar, biomassa fresca e seca da parte aérea, e conteúdo de reservas, superiores às plantas não inoculadas. A eficiência da simbiose foi maior com os isolados oriundos de pomares de pessegueiros, quando comparados aos isolados oriundos de vinhedos.

Termos para indexação: Glomus clarum, Glomus etunicatum, propagação de plantas.

\section{SEEDLINGS DEVELOPMENT OF PEACH 'OKINAWA' INOCULATED WITH ARBUSCULAR MYCORRHIZAL ISOLATED FROM PEACH ORCHARDS AND VINEYARDS}

\begin{abstract}
This study aimed to evaluate the influence of the species of arbuscular mycorrhizal (AM) Glomus clarum and Glomus etunicatum species isolated of peach orchards and vineyard on the vegetative growth, content of nutrients and carbohydrate contents on peach rootstocks cv. Okinawa. The experiment was conducted in a greenhouse, with a split-plot experiment, with 15 plants per plots and four repetitions. AM inoculation favored the absorption of nitrogen, phosphorus and potassium, contributing so that the plants presented the largest answers in height, diameter, foliate area, fresh and dry mass of the aerial part, and carbohydrate contents, superiors to non inoculated plants. The efficiency of the symbiosis was bigger with isolated deriving of peach tree orchards, when compared with isolated deriving of grapevine orchards. Index terms: Glomus clarum, Glomus etunicatum, plant propagation.
\end{abstract}

\section{INTRODUÇÃO}

A eficiência da simbiose é representada pela capacidade das micorrizas arbusculares (MA) em estimular o crescimento da planta e é dependente da genética do fungo e do hospedeiro e das respostas diferenciadas das espécies de MA aos fatores climáticos e às características químicas e físicas do solo (NUNES et al., 2011). Desta forma, têm sido relatadas diferenças entre as espécies de MA na promoção do crescimento e do desenvolvimento de uma mesma espécie vegetal, sendo interpretadas como especificidade (STÜRMER; SIQUEIRA, 2011) ou compatibilidade funcional entre o hospedeiro e o fungo (SCHREINER, 2007). Herrera-Medina et al. (2007) observaram que esta especificidade funcional está relacionada com o papel que os hormônios vegetais, especialmente o ácido abscísico (ABA), desempenham durante o estabelecimento da simbiose. Estes autores relataram que o ABA contribui para que a espécie vegetal tenha, ou não, suscetibilidade à infecção do FMA, em função de seu importante papel, relacionado com o desenvolvimento de um arbúsculo completo e funcional.

\footnotetext{
1(Trabalho 265-12). Recebido em: 29-10-2012. Aceito para publicação em: 11-07-2013.

${ }^{2}$ Engenheiro Agrônomo, Doutor em Fitotecnia, BADESUL desenvolvimento - Agência de Fomento do Estado do Rio Grande do Sul. Rua Silvio Silveira Soares, 2406/130 - Bairro Camaquã - Porto Alegre, RS, CEP 91910-460. Autor para correspondência. E-mail: jose.nunes@badesul.com.br

${ }^{3}$ Engenheiro Agrônomo, Doutor, Professor da Universidade Federal do Rio Grande do Sul, Faculdade de Agronomia, Departamento de Horticultura e Silvicultura. E-mail: pvdsouza@ufrgs.br, marodin@ufrgs.br.

${ }^{4}$ Engenheiro Agrônomo, Doutor, Professor Titular do Departamento de Fitotecnia, Faculdade de Agronomia Eliseu Maciel, Universidade Federal de Pelotas. E-mail: jfachi@ufpel.edu.br.
} 
Wehner et al. (2009) observam que o aumento da absorção de nutrientes e do equilíbrio do estado nutricional das plantas promovido pela ação das MAs podem influenciar no crescimento das plantas, além de promover o aumento da tolerância a estresses abióticos. Desta forma, se por um lado as hifas e o micélio externo aumentam a capacidade de exploração do solo pela raiz, promovendo maior absorção de nutrientes (ANZANELLO et al., 2011), por outro o favorecem na relação água-planta, permitindo maior resistência das plantas às condições de déficit hídrico, em decorrência de uma série de mudanças que ocorrem em sua fisiologia (ALLEN, 2007).

Segundo Purin et al. (2006), a dinâmica das populações de MA presentes em uma área é fundamental para se entender sua influência sobre as condições de estabelecimento, desenvolvimento e manutenção de uma população de plantas neste local. Além disto, a diversidade, a densidade e o potencial de infectividade dos propágulos de MA no solo estão relacionados indiretamente com as condições climáticas e edáficas de cada ecossistema (SCHREINER, 2007) e diretamente com a fisiologia do fungo (POUYU-ROJAS et al., 2006; STÜRMER; SIQUEIRA, 2011), estando a colonização micorrízica ligada ao genótipo da planta e do fungo, assim como ao ambiente. Neste aspecto, as espécies de MA autóctones de uma determinada região, que já são adaptadas às condições edafoclimáticas da mesma, tendem a conferir maior potencial de resposta, quando inoculadas nas plantas a serem cultivadas nesta região (ANZANELLO et al., 2011).

O objetivo deste trabalho foi avaliar a eficiência de isolados das espécies de MA Glomus clarum e Glomus etunicatum oriundas de pomares de pessegueiros da região da Depressão Central e de vinhedos da Serra Gaúcha, sobre o crescimento vegetativo, conteúdo de macronutrientes e de substâncias de reserva de plantas do porta-enxerto de pessegueiro cv. Okinawa.

\section{MATERIAL E MÉTODOS}

O experimento foi conduzido em telado (50\% de sombreamento) localizado na Estação Experimental Agronômica da Universidade Federal do Rio Grande do Sul (EEA-UFRGS), município de Eldorado do Sul-RS, localizada à latitude $30^{\circ} 05^{\prime}$ sul e longitude $51^{\circ} 39^{\prime}$ oeste, durante um período de 10 meses, a partir de 02 de setembro de 2005.

Caroços do porta-enxerto cv. Okinawa foram estratificados em recipientes (caixa plástica de $40 \mathrm{~cm}$ X $28 \mathrm{~cm} \mathrm{X} 10 \mathrm{~cm}$ ) contendo areia e colocados, por um período de 45 dias, em refrigerador, à temperatura de $4^{\circ} \mathrm{C}$, para interromper a dormência do embrião e promover a germinação. A areia foi previamente autoclavada a $120^{\circ} \mathrm{C}$ por uma hora. Após o período de estratificação, as sementes foram retiradas dos caroços e semeadas em caixas plásticas $(40 \mathrm{~cm} \mathrm{X}$ $28 \mathrm{~cm} \mathrm{X} 10 \mathrm{~cm}$ ), preenchidas com areia autoclavada e mantidas em casa de vegetação, equipada com sistema de irrigação por aspersão. Nos primeiros 60 dias, o intervalo do turno de irrigação era de 24 horas, no início do dia. Nos meses mais quentes (novembro, dezembro, janeiro, fevereiro e março), o número de turnos foi aumentado para dois, de 12 horas de intervalo, feitos no início e no final do dia. Ao final do experimento, o intervalo do turno de irrigação voltou a ser de 24 horas, no início do dia.

Quando as plântulas apresentavam cerca de $5 \mathrm{~cm}$ de altura da parte aérea, foram repicadas para sacos plásticos pretos (5 litros), contendo substrato constituído de terra argilosa de um solo Argissolo Vermelho distrófico típico (VEZZANI; MIELNICZUK, 2011), areia (granulometria média, entre 0,6 e $1 \mathrm{~mm}$ ) e resíduo decomposto de casca de acácia negra $(1: 1: 1, \mathrm{~V}: \mathrm{V} . \mathrm{V})$. O substrato foi previamente desinfestado visando à eliminação de microrganismos presentes neste, utilizando-se de solução de formaldeído, a $10 \%$.

Imediatamente antes da repicagem, procedeuse à adição dos inóculos de MA ao substrato (30 gramas por saco plástico, 10 esporos por grama de inóculo), em uma camada situada na altura intermediária de cada recipiente (aproximadamente a $15 \mathrm{~cm}$ de altura). As espécies de MA testadas foram Glomus clarum (NICOL. \& SCHENCK) e Glomus etunicatum (BECKER \& GERD), cujos esporos foram isolados de amostras de solo retirados de vinhedos da Serra Gaúcha e de pomares de pessegueiro da região da Depressão Central, através do método de lavagem, decantação, peneiramento e centrifugação (STÜRMER; SIQUEIRA, 2011). A identificação morfológica das espécies foi realizada através da observação em microscópio óptico (KIVLIN et al., 2011). A multiplicação das espécies foi realizada por cultivo monospórico, utilizando-se das espécies vegetais orégano (Origanum vulgare Link), para os isolados de vinhedos, e braquiária (Brachiaria decumbens Stapf.), para os isolados de pomares de pessegueiro. O delineamento experimental utilizado foi o de blocos casualizados, em esquema de parcelas subdivididas (a origem das MAs constituiu a parcela principal, e a espécie, a subparcela), com 15 plantas por parcela e quatro repetições.

Aos 300 dias após a inoculação, foi feita a avaliação da altura das plantas, desde o colo até o ápice da haste principal, e do diâmetro da haste 
principal, na altura do colo das plantas. Para tanto, foi utilizada uma trena e um paquímetro da marca RS Baty. Além disto, 5 plantas de cada repetição dos tratamentos foram usadas para a determinação da área foliar, através do uso de medidor de área foliar marca Li-Cor (modelo LI - 3000), e da biomassa fresca e seca da parte aérea, através de pesagem e secagem até peso constante. As amostras secas foram moídas e delas foram retiradas frações para avaliação de conteúdo de macronutrientes por digestão, destilação e espectrofotometria de chamas do tecido vegetal (VEZZANI; MIELNICZUK, 2011).

Das amostras moídas, foram coletadas subamostras de um grama cada, que foi acondicionada em saquinho feito com tela especial para filtragem e digestão, anotando-se os pesos de cada saquinho antes e após processo de digestão em solução aquosa, com 5\% de ácido tricloroacético (99\%) e $35 \%$ de metanol (99\%), visando à extração de todos os componentes do tecido vegetal convencionados como substâncias de reserva (carboidratos, gorduras, etc.) que não fossem fibras (celulose, hemicelulose e lignina) (ANZANELLO et al., 2011). Além disto, foram coletados segmentos de raízes secundárias, com $1 \mathrm{~cm}$, para determinar a colonização radicular (número de segmentos infectados/total analisado) e índices de presença de hifas ( 0 - ausência; 1 - fraca; 2 - moderada; 3 - intensa), vesículas e arbúsculos (0: ausência; 1: 1 a 50; 2: 51 a 100; 3: mais de 100 estruturas/cm de radicela) (KIVLIN et al., 2011).

Os dados foram submetidos à análise de variância, pelo programa SAS, e as médias foram comparadas pelo teste de Duncan, ao nível de 5\% de significância. Foram feitas análises de correlação simples, empregando-se o coeficiente de correlação de Pearson (r) com valores máximos representados por $\mathrm{r}=1$ e $\mathrm{r}=-1$.

\section{RESULTADOS E DISCUSSÃO}

As espécies de MA aceleraram o crescimento das plantas 'Okinawa', induzindo maior altura, diâmetro, área foliar, biomassa fresca e seca dos tecidos da parte aérea, quando comparadas com a testemunha (Tabela 1). O isolado de G. etunicatum oriundo de pomares de pessegueiros proporcionou os maiores incrementos em todos os parâmetros de desenvolvimento vegetativo, enquanto o isolado de G. clarum de mesma origem promoveu ganhos em altura, diâmetro do caule e área foliar superior aos isolados de G. clarum e G. etunicatum originados de vinhedos, apresentando, porém, resultados de biomassa fresca e seca semelhante a estes. Os isolados de G. clarum e G. etunicatum oriundos de vinhedos proporcionaram às plantas incrementos dos parâmetros de crescimento avaliados semelhantes entre si, superiores às testemunhas.

$\mathrm{O}$ fato de as MAs causarem desenvolvimento vegetativo diferenciado, quando inoculadas em frutíferas, está associado à afinidade, maior ou menor, que cada espécie de MA tem com a espécie vegetal utilizada, que estaria relacionada à existência de uma compatibilidade funcional entre o hospedeiro e o fungo (SCHREINER, 2007). Porém, os resultados obtidos mostraram que os isolados de ambas as espécies, oriundos dos vinhedos, apresentavam compatibilidade funcional com a cultivar semelhante, enquanto o isolado de G. etunicatum, oriundo de pomares de pessegueiros, mostrou maior afinidade com as plantas da cv. Okinawa, apresentando maiores resultados do que o isolado de G. clarum de mesma origem (Tabela 1).

O incremento em crescimento, proporcionado pelas MAs, pode variar em função da espécie utilizada e do isolado de uma mesma espécie de MA(HIPPLER et al., 2011). Os mesmos autores observam que MAs autóctones de determinado ambiente podem estar mais adaptadas às condições prevalecentes, sendo mais efetivas que espécies introduzidas. Este relato vem ao encontro dos resultados obtidos com as espécies isoladas de pomares de pessegueiros e de vinhedos, e da cultivar de porta-enxerto utilizada no experimento. Os resultados referentes ao crescimento vegetativo são coincidentes com os obtidos por Silva Júnior et al. (2012), que, trabalhando com plantas de meloeiro inoculadas com as espécies G. clarum e G. etunicatum oriundos de pomares de meloeiros, observaram aumentos de altura e de diâmetro, quando feita a comparação às testemunhas, apresentando, também, crescimento superior a plantas inoculadas com a mesma espécie de MA isolada de áreas de lavoura.

A área foliar é um importante parâmetro, por definir a taxa de fotossíntese realizada na planta (NUNES et al., 2011). Para Vitorazi filho et al. (2012), a simbiose planta-MA proporciona aumento na área foliar em relação a plantas não colonizadas, o que pode explicar os resultados observados em ambos os experimentos (Tabela 1). Associada ao aumento da área foliar, a colonização por MA proporcionaria, também, maior biomassa fresca em plantas inoculadas do que em plantas não inoculadas, em virtude de um maior conteúdo de água, podendo mostrar, porém, biomassa seca semelhante (CARDOSO; KUYPER, 2006; PURIN et al., 2006). Entretanto, as MAs propiciaram os resultados relativos à biomassa seca superiores às testemunhas, o que pode ser atribuído à maior área foliar, que proporcionou maior produção 
de fotoassimilados e acúmulo de biomassa seca.

Vitorazi filho et al. (2012) observaram que o uso de isolados de espécies de MA nativas de pomares de maracujazeiro proporcionaram maior incremento em área foliar e maior biomassa fresca e seca do que isolados das mesmas espécies de MA não nativas. Tais relatos coincidem com os dados obtidos neste trabalho somente para a espécie G. etunicatum, já que os isolados da espécie recuperados de pomares de pessegueiros apresentaram maiores incrementos em área foliar e biomassa fresca e seca do que os isolados da mesma espécie recuperados de amostras de solo de vinhedos, enquanto os resultados obtidos com os isolados de G. clarum, de ambas as áreas, apresentaram resultados semelhantes.

As plantas inoculadas com os isolados de MA apresentaram os maiores percentuais de macronutrientes nos tecidos vegetais da parte aérea, exceção feita ao cálcio e ao magnésio, onde todas as espécies de MA proporcionaram resultados inferiores às plantas- testemunha (Tabela 2). Os isolados de $G$. clarum e G. etunicatum oriundos de vinhedos proporcionaram às plantas inoculadas percentuais semelhantes para todos os macronutrientes, enquanto o isolado de G. etunicatum, originário de pomares de pessegueiros, propiciou os maiores percentuais de nitrogênio, fósforo e potássio, quando comparado ao isolado de $G$. clarum de mesma procedência. Conforme observam Anzanello et al. (2011), as MAs induzem maior absorção destes elementos, sendo de vital importância para as plantas. Segundo Purin et al. (2006), através de mecanismos promovidos pelas MAs, as hifas e o micélio externo aumentam a capacidade de exploração do solo pela raiz, o que proporciona maior absorção de nutrientes. A eficiência das hifas deve-se ao seu pequeno diâmetro e grande ramificação no solo, podendo aumentar a superfície de absorção das raízes em até 700\% (OZDEMIR et al., 2010). Com relação aos macronutrientes cálcio e magnésio, Herrera-Medina et al. (2007) afirmam que estes são vitais para o desenvolvimento das plantas, por participarem da regulação da hidratação e da ativação de enzimas, além de participarem da fotossíntese, no caso do magnésio. Porém, segundo observaram Anzanello et al. (2011), a diminuição da concentração de cálcio e magnésio em plantas infectadas pode ser devida à sua diluição nos tecidos, em função do incremento no crescimento vegetativo das plantas colonizadas, ou à capacidade das MAs em reduzir a absorção desses elementos, em função de um efeito tampão proporcionado pelos fungos (NUNES et al., 2011), o que está em concordância com os resultados obtidos neste trabalho.

Verifica-se, também, que as plantas inocu- ladas com o intervalo do turno de irrigação de MA apresentaram os maiores teores de substâncias de reserva (carboidratos, gorduras, ácidos graxos, etc.) dos tecidos da parte aérea, superiores às plantas-testemunha (Tabela 2). Plantas com maior altura e área foliar apresentam maior capacidade de captação de luz e produção de fotoassimilados, o que permite um fluxo mais intenso de carboidratos no sentido radicial, onde uma parte seria utilizada pelas MAs em sua nutrição e acumulação em estruturas de reserva (vesículas), e o restante seria acumulado nos tecidos de armazenamento da planta, na forma de substâncias de reserva (OZDEMIR et al., 2010).

Todos os tratamentos com MAs apresentaram altas taxas de colonização radicular, acima de 90\% (Tabela 3). O índice de colonização das raízes com hifas, vesículas e arbúsculos foi considerado de fraco a médio para todas as espécies de MA, sendo que só ocorreu diferença entre os isolados de G. etunicatum e G. clarum oriundos de pomares de pessegueiros, com índices superiores em plantas inoculadas com a primeira espécie. Estes resultados de colonização radicular indicam a maior eficiência da simbiose do isolado e G. etunicatum oriundo de pomares de pessegueiro com o porta-enxerto cv. Okinawa, nas condições em que foram realizados os experimentos.

Ao avaliar o grau de associação entre os diversos parâmetros de crescimento e nutrição mineral da cv. Okinawa com a colonização radicular pelos isolados de MA no experimento, verificou-se que houve correlação entre os mesmos, ou seja, muitas das variáveis avaliadas estão associadas, positiva ou negativamente, com o percentual de colonização radicular, independentemente da espécie de MA utilizada (Tabela 4). As correlações positivas foram consideradas muito significativas $(\mathrm{P}<0,01)$ entre o percentual de colonização das raízes e a altura da planta, o diâmetro do caule, a biomassa fresca e seca da parte aérea, o percentual de nitrogênio, fósforo e de potássio na parte aérea e substâncias de reserva na parte aérea para ambos os isolados, e entre o percentual de colonização das raízes e a área foliar dos isolados originados de pomares de pessegueiros. Foram consideradas significativas $(\mathrm{P}<0,05)$ as correlações positivas entre o percentual de colonização das raízes e a área foliar dos isolados originados de vinhedos. As correlações negativas foram consideradas muito significativas entre o percentual de colonização das raízes e o percentual de cálcio, e significativas para o percentual de magnésio da parte aérea, tanto para os isolados de vinhedos como para os de pomares de pessegueiro. Isto indica que, quanto maior a colonização radicular, menor a absorção destes elementos, em função de um efeito tampão proporcionado pelas 
MAs (ANZANELLO et al., 2011), variando em função da espécie de fungo inoculado e da espécie vegetal utilizada (CARDOSO; KUYPER, 2006).

Diversos autores observaram correlações significativas entre as respostas de crescimento das plantas, percentagem de colonização e conteúdo de nutrientes, como, por exemplo, Vitorazi Filho et al. (2012), trabalhando com plantas de maracujazeiro-doce, e Silva Júnior et al. (2012), que trabalharam com mudas de meloeiro. Costa et al. (2005), trabalhando com plantas de mangabeira, observaram correlações significativas entre o percentual de colonização e o conteúdo de nutrientes, especialmente fósforo. Porém, estes autores observam que pode existir o efeito negativo do aumento da disponibilidade do fósforo no solo sobre a eficiência da simbiose micorrízica, fato não observado no presente estudo.

$\mathrm{O}$ aumento da taxa fotossintética de plantas inoculadas com MA está diretamente relacionado ao aumento da área foliar, o que proporciona aumento do crescimento vegetativo e acúmulo de biomassa fresca e seca (SILVA JÚNIOR et al., 2012). Além disto, segundo observam Wright et al. (2005), plantas mais altas e com maior área foliar apresentam maior capacidade de fotossíntese e produção de fotoassimilados, proporcionando acúmulo de biomassa e, por conseguinte, maior nível de carbono assimilado. Isto porque, plantas com maior altura e área foliar apresentam maior capacidade de captação de luz e produção de fotoassimilados, o que permite um fluxo mais intenso de carboidratos no sentido radicular, onde uma parte seria utilizada pelas MAs em sua nutrição e acumulação em estruturas de reserva (vesículas), e o restante seria acumulado nos tecidos de armazenamento da planta, na forma de substâncias de reserva (OZDEMIR et al., 2010). Cabe salientar também que um maior diâmetro de caule, que seria proporcionado pelas MAs, permitiria um aumento do fluxo ascendente de água e de nutrientes, e de seiva elaborada, no sentido descendente (ALLEN, 2007; CARRENHO et al., 2007).

Tais relatos coincidem com os resultados obtidos com os isolados das espécies de MA testadas, principalmente no caso das plantas inoculadas com isolados de pomares de pessegueiros, onde o isolado de G. etunicatum apresentou os maiores resultados em termos de altura, diâmetro e área foliar, apresentando, seguidos pelo isolado de G. clarum de mesma origem, com comportamento intermediário, enquanto os isolados das mesmas espécies, originárias de vinhedos, apresentaram comportamento superior somente às plantas não inoculadas.

Maiores estudos devem ser conduzidos para avaliar o efeito da diminuição do conteúdo de cálcio e magnésio nos tecidos sobre a longevidade pós-colheita dos frutos.

TABELA 1- Altura, diâmetro, área foliar, biomassa fresca e seca de folhas e hastes de plantas da cv. Okinawa inoculadas com isolados de duas espécies de micorriza arbuscular (MA) (Glomus clarum e G.etunicatum), oriundos de pomares de pessegueiros e de vinhedos, medidos aos 300 dias após a inoculação. Eldorado do Sul-RS, 2006.

\begin{tabular}{|c|c|c|c|c|c|c|}
\hline \multirow{4}{*}{ Origem do isolado } & \multirow{4}{*}{ MA } & \multirow{3}{*}{ Altura } & \multirow{3}{*}{ Diâmetro } & \multirow{3}{*}{ Área foliar } & \multicolumn{2}{|c|}{ Parte aérea } \\
\hline & & & & & \multicolumn{2}{|c|}{ Biomassa } \\
\hline & & & & & Fresca & Seca \\
\hline & & $(\mathrm{cm})$ & $(\mathrm{mm})$ & $\left(\mathrm{cm}^{2} /\right.$ planta $)$ & (g) & (g) \\
\hline \multirow{2}{*}{ Pomares de pessegueiro } & G. clarum & $110,92 b^{1}$ & $6,54 \mathrm{~b}$ & $762,32 b$ & $165,00 \mathrm{~b}$ & $107,00 \mathrm{~b}$ \\
\hline & G. etunicatum & $123,65 \mathrm{a}$ & $7,72 \mathrm{a}$ & $891,71 \mathrm{a}$ & $197,00 \mathrm{a}$ & $129,00 \mathrm{a}$ \\
\hline \multirow{2}{*}{ Vinhedos } & G. clarum & $99,73 \mathrm{c}$ & $5,93 \mathrm{c}$ & $764,31 b$ & $163,00 \mathrm{~b}$ & $115,00 \mathrm{~b}$ \\
\hline & G. etunicatum & $103,80 \mathrm{c}$ & $5,93 \mathrm{c}$ & $739,82 b$ & $167,00 \mathrm{~b}$ & $115,00 \mathrm{~b}$ \\
\hline Testemunha & - & $92,04 d$ & $5,06 \mathrm{~d}$ & $537,27 \mathrm{c}$ & $136,50 \mathrm{c}$ & $84,00 \mathrm{c}$ \\
\hline$\overline{\mathrm{CV}(\%)}$ & & 6,21 & 7,81 & 8,76 & 6,97 & 7,01 \\
\hline
\end{tabular}

${ }^{1}$ Médias seguidas de letras distintas, na coluna, diferem entre si, pelo teste Duncan $(\mathrm{p} \leq 0,05)$. 
TABELA 2- Conteúdo de macronutrientes (\%) e substâncias de reserva (\%) encontrados na parte aérea (folhas e hastes) de plantas do porta-enxerto de pessegueiro cv. Okinawa inoculadas com isolados de duas espécies de micorriza arbuscular (MA) (Glomus clarum e G.etunicatum), oriundos de pomares de pessegueiro e de vinhedos, medidos aos 300 dias após a inoculação. Eldorado do Sul-RS, 2006.

Origem do isolado MA

$\mathrm{N} \quad \mathrm{P} \quad \mathrm{K} \quad \mathrm{Ca} \quad \mathrm{Mg} \quad$ Substâncias de reserva

\begin{tabular}{|c|c|c|c|c|c|c|c|}
\hline \multirow{3}{*}{$\begin{array}{l}\text { Pomares de } \\
\text { pessegueiro }\end{array}$} & \multirow{3}{*}{$\begin{array}{l}\text { G. clarum } \\
\text { G. etunicatum }\end{array}$} & \multicolumn{6}{|c|}{ \% Parte aérea } \\
\hline & & $3,08 b^{1}$ & $0,19 b$ & $2,29 b$ & $1,24 \mathrm{~b}$ & $0,40 \mathrm{~b}$ & $36,03 \mathrm{a}$ \\
\hline & & $3,41 \mathrm{a}$ & $0,24 \mathrm{a}$ & $2,49 \mathrm{a}$ & $1,23 b$ & $0,41 b$ & $35,81 \mathrm{a}$ \\
\hline \multirow{2}{*}{ Vinhedos } & G. clarum & $2,23 \mathrm{c}$ & $0,15 \mathrm{c}$ & $1,74 \mathrm{c}$ & $1,32 b$ & $0,41 b$ & $35,05 \mathrm{a}$ \\
\hline & G. etunicatum & $2,22 \mathrm{c}$ & $0,15 \mathrm{c}$ & $1,82 \mathrm{c}$ & $1,30 \mathrm{~b}$ & $0,43 b$ & $35,53 \mathrm{a}$ \\
\hline \multirow{2}{*}{ Testemunha } & - & $2,06 \mathrm{~d}$ & $0,13 d$ & $1,61 d$ & $1,50 \mathrm{a}$ & $0,57 \mathrm{a}$ & $21,14 b$ \\
\hline & & 8,12 & 7,20 & 7,71 & 6,44 & 5,31 & 5,56 \\
\hline
\end{tabular}

${ }^{1}$ Médias seguidas de letras distintas, na coluna, diferem entre si, pelo teste Duncan $(\mathrm{p} \leq 0,05)$.

TABELA 3 - Colonização radicular e presença de estruturas (hifas, vesículas e arbúsculos) em raízes de plantas do porta-enxerto de pessegueiro cv. Okinawa inoculadas com isolados de duas espécies de micorriza arbuscular (MA) (Glomus clarum e G.etunicatum), oriundos de pomares de pessegueiro e de vinhedos, medidos aos 300 dias após a inoculação. Eldorado do Sul-RS, 2006.

\begin{tabular}{|c|c|c|c|c|c|}
\hline \multirow{3}{*}{ Origem do isolado } & \multirow{3}{*}{ MA } & \multirow{2}{*}{ Colonização } & \multicolumn{3}{|c|}{ Índice de presença de estruturas de MA } \\
\hline & & & Hifas $^{2}$ & Vesículas $^{3}$ & Arbúsculos $^{3}$ \\
\hline & & $(\%)$ & & & \\
\hline \multirow{2}{*}{ Pomares de pessegueiro } & G. clarum & $91,00 \mathrm{a}^{1}$ & $1,42 b$ & $1,01 \mathrm{~b}$ & $1,21 b$ \\
\hline & G. etunicatum & $93,00 \mathrm{a}$ & $1,52 \mathrm{a}$ & $1,30 \mathrm{a}$ & $1,47 \mathrm{a}$ \\
\hline \multirow{2}{*}{ Vinhedos } & G. clarum & $91,76 a$ & $1,49 \mathrm{a}$ & $1,26 \mathrm{a}$ & $1,44 a$ \\
\hline & G. etunicatum & $92,62 \mathrm{a}$ & $1,50 \mathrm{a}$ & $1,29 \mathrm{a}$ & $1,46 \mathrm{a}$ \\
\hline Testemunha & - & $0,00 \mathrm{~b}$ & $0,00 \mathrm{c}$ & $0,00 \mathrm{c}$ & $0,00 \mathrm{c}$ \\
\hline CV (\%) & & 2,42 & 8,26 & 8,12 & 8,21 \\
\hline
\end{tabular}

${ }^{1}$ Médias seguidas pela mesma letra, na coluna, não diferem entre si, pelo teste de Duncan, ao nível de 5\% de significância. 2Índice de presença de hifas de FMA: 0: ausência de estruturas; 1: presença fraca; 2: presença moderada; 3: presença intensa.

3Índice de presença de vesículas ou arbúsculos de FMA: 0: ausência de estruturas; 1: 1 a 50 estruturas; 2: 51 a 100 estruturas; 3: mais de 100 estruturas por centímetro de radicela.

TABELA 4 - Correlação (r) entre parâmetros de crescimento (altura, diâmetro, área foliar, biomassa fresca e seca), nutrição mineral (\% de macronutrientes) e substâncias de reserva (\%) da parte aérea (folhas e hastes) de plantas da cv. Okinawa e colonização radicular (\%) por isolados de duas espécies de micorriza arbuscular (MA) (Glomus clarum e G.etunicatum) oriundas de pomares de pessegueiro e de vinhedos, medidos aos 300 dias após a inoculação. Eldorado do Sul-RS, 2006.

Parâmetros

\% Colonização $\mathrm{x}$ altura $\mathrm{r}$

\% Colonização x diâmetro Vinhedos Pomares de pessegueiro

$\%$ Colonização x área foliar

$0,63^{* *}$

$0,82^{* *}$

$0,72 * *$

$0,76^{* *}$

\% Colonização x biomassa fresca

$0,53^{*}$

$0,65 * *$

$\%$ Colonização x biomassa seca

$0,62^{* * *}$

$0,63^{* *}$

$\%$ Colonização x \% nitrogênio

$0,60^{* *}$

$0,64 * *$

$\%$ Colonização x \% fósforo

$0,82 * *$

$0,89 * *$

$\%$ Colonização $\mathrm{x} \%$ potássio

$0,72 * *$

$0,74 * *$

$\%$ Colonização x \% Cálcio

$0,66^{* *}$

$0,74 * *$

\% Colonização x \% Magnésio

$-0,71^{* *}$

$-0,78 * *$

\% Colonização x \% substâncias de reserva

$-0,51$ *

$-0,49$ *

* e **Significativo a $5 \%$ e a $1 \%$ de probabilidade, respectivamente, pelo teste de Duncan. 


\section{CONCLUSÕES}

1-O uso das micorrizas arbusculares, em vaso com substrato tratado com formaldeído a $10 \%$, beneficia as plantas do porta-enxerto de pessegueiro cv. Okinawa, acelerando seu desenvolvimento vegetativo e melhorando seu conteúdo de nitrogênio, fósforo e potássio.

2-A eficiência da simbiose é maior com os isolados oriundos de pomares de pessegueiros.

\section{REFERÊNCIAS}

ALLEN, M.F. Mycorrhizal fungi: highways for water and nutrients in arid soils. Vadose Zone Journal, Madison, v. 6, n. 1, p. 291-297, 2007.

ANZANELLO, R.; SOUZA, P.V.D.; CASAMALI, B. Fungos micorrízicos arbusculares (FMA) em porta-enxertos micropropagados de videira. Bragantia, Campinas, v. 70, n. 2, p. 409-415, 2011.

CARDOSO, I.M.; KUYPER, T.W. Mycorrhizas and tropical soil fertility. Agriculture, Ecosystems \& Environment, Amesterdam, v. 116, n. 1/2, p.72-84, 2006.

CARRENHO, R.; TRUFEM, S.F.B.; BONONI, V.L.R.; SILVA, E.S. The effect of different soil properties on arbuscular mycorrhizal colonization of peanuts, sorghum and maize. Acta Botanica Brasilica, São Paulo, v. 21, n. 3, p. 723-730, 2007.

COSTA, C.M.C.; CAVALCANTE, U.M.T.; GOTO, B.T.; SANTOS, V.F.; MAIA, L.C. Fungos micorrízicos arbusculares e adubação fosfatada em mudas de mangabeira. Pesquisa Agropecuária Brasileira, Brasília, v. 40, n. 3, p. 225-232, 2005.

HERRERA-MEDINA, J.M.; STEINKELLNER, S.; VIERHEILIG, H.; BOTE, J.A.O.; GARRIDO, J.M.G. Abscisic acid determines arbuscule development and functionality in the tomato arbuscular mycorrhiza. New Phytologist, Cambridge, v. 175, n. 3, p. 554564, 2007.

HIPPLER, I.W.R.; MOREIRA, M.; DIAS, N.M.S.; HERMANN, E.R. Fungos micorrízicos arbusculares nativos e doses de fósforo no desenvolvimento de amendoim RUNNER IAC 886. Revista Ciência Agronômica, Fortaleza, v. 42, n. 3, p. 605-610, 2011.
KIVLIN, S.N.; HAWKES, C.V.; TRESEDER, K.K. Global diversity and distribution of arbuscular mycorrhizal fungi. Soil Biology and Biochemistry, Amsterdam, v. 43, n. 11, p. 2294-2303, 2011.

NUNES, J.L.S.; SOUZA, P.V.D.; MARODIN, G.A.B.; FACHINELLO, J.C. Incremento no desenvolvimento do porta-enxerto de pessegueiro 'Okinawa' por fungos micorrízicos arbusculares autóctones. Revista Ceres, Viçosa, MG, v.58, n. 2, p.223-231, 2011.

OZDEMIR, G.; AKPINAR, G.; SABIR, A.; BILIR, H.; TANGOLAR, S.; ORTAS, I. Effect of inoculation with mycorrhizal fungi on growth and nutrient uptake of grapevine genotypes (Vitis spp.). European Journal of Horticultural Science, Stuttgart, v.75, p.103-110, 2010.

POUYU-ROJAS, E.; SIQUEIRA, J.O.; SANTOS, J.G.D. Compatibilidade simbiótica de fungos micorrízicos arbusculares com espécies arbóreas tropicais. Revista Brasileira de Ciência do Solo, Viçosa, MG, v. 30, n. 3, p. 413-424, 2006.

PURIN, S.; KLAUBERG FILHO, O.; STÜRMER, S.L. Mycorrhizae activity and diversity in conventional and organic apple orchards from Brazil. Soil Biology and Biochemistry, Amsterdam, v. 38, n. 7, p. 1831-1839, 2006.

SCHREINER, R.P. Effects of native and nonnative arbuscular mycorrhizal fungi on growth and nutrient uptake of "Pinot noir" (Vitis vinifera L.) in two soils with contrasting levels of phosporus. Applied Soil Ecology, Amsterdam, v. 36, n. 2/3, p. 205-215, 2007.

SILVA JÚNIOR, J.M.T.; MENDES FILHO, P.F.; GOMES, V.F.F.; GUIMARÃES, F.V.A.; SANTOS, E.M. Efeito da esterilização do substrato sobre o crescimento de mudas de meloeiro em presença de fungos micorrízicos arbusculares e compostos orgânicos. Revista Caatinga, Mossoró, v. 25, n. 1, p. 98-103, 2012.

STÜRMER, S.L.; SIQUEIRA, J.O. Species richness and spore abundance of arbuscular mycorrhizal fungi across distint land uses in Western Brazilian Amazon. Mycorrhiza, Adelaide, v. 21, n. 4, p. 255-267, 2011. 
VITORAZI FILHO, J.A.; LIMA, K.B.; FREITAS, M.S.M.; MARTINS, MA.A.; OLIVARES, F.L. Crescimento de mudas de maracujazeiro-doce inoculadas com fungos micorrízicos arbusculares e bactérias diazotróficas sob diferentes doses de fósforo. Revista Brasileira de Fruticultura, Jaboticabal, v. 34, n. 2, p. 442-450, 2012.

VEZZANI, F.M.; MIELNICZUK, J. Agregação e estoque de carbono em Argissolo submetido a diferentes práticas de manejo agrícola. Revista Brasileira de Ciência do Solo, Viçosa, MG, v. 35, n. 1, p. 213-223, 2011.
WEHNER, J.; ANTUNES, P.M.; POWELL, J.R.; MAZUKATOW, J.; RILLIG, M.C. Plant pathogen protection by arbuscular mycorrhizas: A role for fungal diversity? Pedobiologia, Berlin, v. 53, n. 3, p. $197-201,2009$.

WRIGHT, D.P.; SCHOLES, J.D.; READ, D.J.; ROLFE, S.A. European and African maize cultivars differ in their physiological and molecular responses to mycorrhizal infection. New Phytologist, Cambridge, v. 167, n. 3, p. 881-896, 2005. 